\title{
EDITORIAL
}

For reprint orders, please contact: reprints@futuremedicine.com

\section{Common pathobiologic origins of chronic obstructive pulmonary disease and lung cancer: more than just a smoking gun}

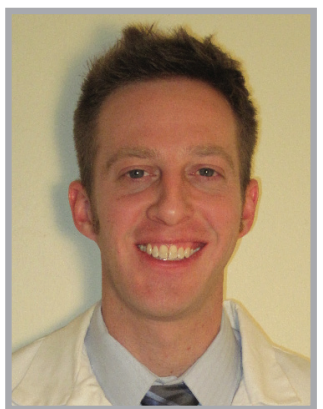

Gregory A Hickey*1

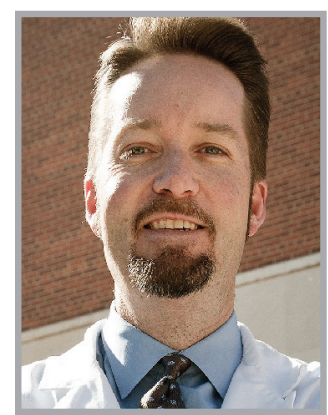

Robert L Keith ${ }^{1,2}$

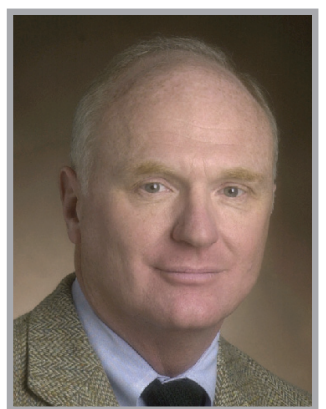

York E Miller ${ }^{1,2}$

"The evidence suggests that four areas in particular contribute

to the shared risk of these two diseases: inflammation, acquired genetic and epigenetic alterations, inheritance and a hypoxic cellular microenvironment."

Chronic obstructive pulmonary disease (COPD) and primary malignancies of the lung are highly prevalent, and often fatal, conditions affecting large numbers of people worldwide. As of 2010, there were approximately 14.8 million cases of COPD that were physician diagnosed in the USA, as well as an additional 12 million estimated undiagnosed cases. There are well over 100,000 deaths annually attributed to COPD in the USA [1]. Lung cancer remains the most lethal malignancy worldwide. It is estimated that lung cancer will account for over 160,000 deaths in the USA alone in 2012 [2]. Tobacco use is a well-publicized - and potent - shared risk factor between the two. There is a large body of evidence suggesting that there are other factors connecting these two disease processes as well. Reduced forced expiratory volume in $1 \mathrm{~s}$, after adjusting for cigarette smoking status, conferred a significantly increased risk of lung cancer [3]. Studies disagree regarding whether there is a linear relationship between the degree of airflow obstruction and the risk of malignancy [4]. The data are also controversial with regards to whether or not computed tomography evidence of emphysema (even in the absence of airflow obstruction) represents an increased risk of primary lung malignancy; one study demonstrated a 3.6fold increase in lung cancer [5]. We will focus on underlying pathways and mechanisms common to both COPD and lung cancer. The evidence suggests that four areas in particular contribute to the shared risk of these two diseases: inflammation, acquired genetic and epigenetic alterations, inheritance and a hypoxic cellular microenvironment.

\section{Inflammation}

While airway inflammation is a wellrecognized hallmark of COPD, its role in the development of lung cancer is not as well understood. There is evidence, however, connecting chronic inflammation (be

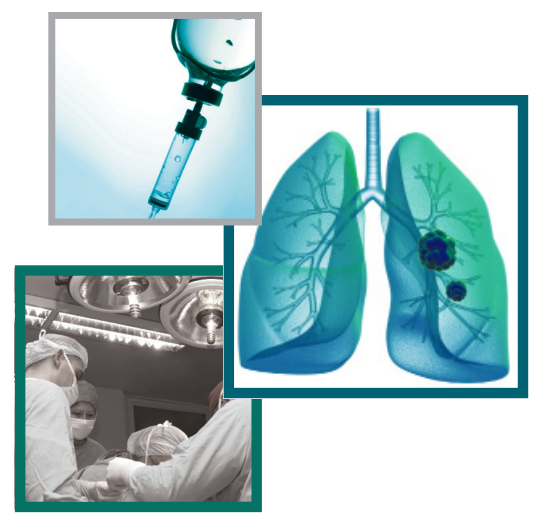

\footnotetext{
"As inflammation may predispose an individual to developing chronic obstructive pulmonary disease or lung cancer, anti-inflammatory therapies have shown utility in a protective or chemopreventive role."
}

'Pulmonary Sciences \& Critical Care Medicine, University of Colorado, Denver, CO, USA

${ }^{2}$ Denver Veteran's Affairs Medical Center, Denver, CO, USA

*Author for correspondence: gregory.hickey@ucdenver.edu

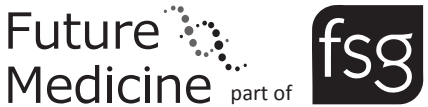


it from long-term tobacco exposure, infection or other mechanisms) to lung cancer. A number of observational studies have previously demonstrated such an association, including an observational cohort of 7081 patients (without known malignancy) who were followed for a 10 -year period. The study demonstrated that subjects with a baseline C-reactive protein level greater than $3.0 \mathrm{mg} / \mathrm{dl}$ were more likely to develop lung cancer (hazard ratio: 2.8; 95\% CI: 1.6-4.9) [6]. There is also a well-described association between TB infection and lung cancer. This was first reported in 1810, but has more recently been described in two large population-based studies in China and Taiwan $[7,8]$. The first study was a retrospective study of 42,422 patients and revealed increased lung cancer mortality (25 vs 3.1 per 1000 patientyears) in the TB-infected patients [7], and was subsequently confirmed by the Taiwanese study [8]. Additionally, a recently published retrospective case series revealed that approximately 25\% of patients that were identified with lung cancer also had a history of Mycobacterium avium complex (MAC) infection (or colonization) [9]. Unlike TB, where it may be easier to establish a relationship between the initial infection and the subsequent development of lung cancer, the temporal sequence between inflammation from MAC infection versus a potentially MAC-friendly tumor microenvironment is not as clearly delineated [10].

\section{"While the pattern of methylation may vary between chronic obstructive pulmonary disease and lung cancer, it would appear that an abnormal methylation pattern in response to long-term tobacco exposure occurs with both."}

As inflammation may predispose an individual to developing COPD or lung cancer, anti-inflammatory therapies have shown utility in a protective or chemopreventive role. A large observational cohort study revealed that patients using inhaled corticosteroids (a commonly accepted treatment modality for inflammatory airway diseases, including COPD) had decreased rates of lung cancer when compared with similar patients not using an inhaled corticosteroid [11]. Subsequent prospective studies have not been as conclusive. Two separate studies of inhaled corticosteroids (one with budesonide and another with fluticasone) on computed tomographydetected lung nodules in high-risk individuals revealed a significant regression in existing target nodules, but a nonsignificant difference in the appearance of new ones [12,13]. One mechanism of inhaled corticosteroid activity is by modulation of the production of PGE2 in the COX-2 pathway [11]. Abnormal eicosanoid metabolism, particularly COX-2 upregulation, has been seen in both carcinoma in situ and non-small-cell lung cancer. Other chemoprevention studies have targeted this pathway as well. Oral iloprost, a prostacyclin analog, significantly improved endobronchial dysplasia in former smokers in a randomized, double-blind study [14].

\section{Genetic \& epigenetic alterations}

Genetic and epigenetic alterations are well known to be important in carcinogenesis, and there is emerging evidence that they may also play a role in COPD. K-ras mutations have been demonstrated to upregulate inflammatory mediators in lung tumors [15]; however, the potential for mutations in premalignant lung tissue to incite inflammation and promote the development of COPD has not been fully explored. Patients with lung cancer and patients with COPD both have abnormal patterns of genome methylation. A study comparing smokers with lung cancer to cancer-free controls (both smokers and non-smokers) revealed promoter hypermethylation at the p16 tumor suppressor locus in patients with lung cancer, as well as the current and former smokers. Only never-smokers had a normal methylation pattern at the p16 locus. The authors concluded that loss of expression of this gene in the bronchial epithelium of smokers was likely permissive for subsequent genetic and epigenetic changes leading to lung cancer [16]. Gene methylation studies have also been conducted in COPD. Unlike the previous study, which utilized DNA from bronchial epithelium, the DNA obtained in a study by Qiu et al. was from peripheral blood, a tissue relevant to systemic inflammation [17]. By contrast, this study detected multiple hypomethylated loci. The upregulated genes encoded at the hypomethylated sites were predominantly involved in immune and inflammatory pathways, consistent with our current understanding of COPD as an inflammatory process. While the pattern of methylation may vary between COPD and lung cancer, it would appear that an abnormal methylation pattern in response to long-term tobacco exposure occurs with both.

There is a host of additional pathways that we have not discussed in detail (e.g., abnormal miRNA expression) [18] by which tobacco 
exposure and chronic inflammation promote both COPD and lung cancer. Each pathway results in genetic and/or epigenetic alteration of the bronchial epithelium. As these alterations accumulate, the epithelial cell undergoes progressive phenotypic change, with each step gradually bringing the cell closer to malignant transformation.

\section{Inheritance}

Multiple epidemiology studies clearly demonstrate inter-related inheritance patterns of COPD, lung cancer and impaired respiratory physiology. Not surprisingly, a primary relative with a history of lung cancer increases risk twofold. Furthermore, a family history of COPD also increases one's risk of developing lung cancer. Similarly, family members of individuals with either COPD or lung cancer are more likely to have an impaired forced expiratory volume in 1 s:forced vital capacity ratio when compared with neighborhood controls and adjusted for age, sex, race and smoking history $[5,19]$.

\section{"Long-term inflammation with resulting phenotypic alteration to the bronchial epithelium, genetic predisposition and \\ a hypoxic microenvironment have each been implicated in pathways shared by chronic obstructive pulmonary disease and lung cancer."}

Most linkage studies investigating the inheritance of COPD and lung cancer have been limited by a relatively small sample size (and therefore larger identified loci). There are potential overlap regions on chromosomes $6 \mathrm{q}$ (with genes involved in both lung function and lung cancer), as well as $12 \mathrm{p}$ (lung function, lung cancer and COPD) [20]. Studies of inherited forms of emphysema have also revealed increased risk of malignancy. Carriers of both the $z$ and $s$ allele for $\alpha 1$ antitrypsin deficiency are at a 2.2-fold increased risk of developing lung cancer (after adjusting for age, gender and COPD history) [6]. One study has also demonstrated an increased risk associated with single-nucleotide polymorphisms at the 15q25 locus, which contains three separate genes encoding for nicotinic acetylcholine receptors that are thought to play a role in nicotine addiction. These singlenucleotide polymorphisms were associated with an increased lung cancer risk, with an odds ratio of 1.3 (corresponding to an equivalent risk of $<0.5$ cigarettes per day) [21].

\section{Hypoxia}

Recently, we have reported evidence that chronic hypoxia, as is often seen within the lungs of patients with severe COPD, may promote lung tumor growth. Recent animal studies have shown that mice exposed to chronic hypoxia in conjunction with a chemical carcinogenesis protocol developed tumors with increased volume compared with normoxic controls. The tumors and lungs from the hypoxic group demonstrated increased expression of the transcription factor HIF-2 $\alpha$, as well as accompanying downstream upregulation of VEGF-A and other molecules involved in survival, proliferation and angiogenesis signaling pathways. Tumors in the hypoxic group did display increased sensitivity to VEGF/EGFR inhibition, suggesting a possible role for targeted chemoprevention or therapy in high-risk (i.e., chronically hypoxic) populations [22].

\section{Conclusion}

An extensive body of evidence suggests a fair degree of commonality between primary lung malignancy and COPD. Long-term inflammation with resulting phenotypic alteration to the bronchial epithelium, genetic predisposition and a hypoxic microenvironment have each been implicated in pathways shared by COPD and lung cancer. Additional novel common mechanisms of pathogenesis, such as genetic or epigenetic epithelial alterations, are emerging and require further investigation. Identification and further understanding of these pathways remains of the utmost importance, as each one may represent an opportunity for treatment or prevention of both of these diseases, or perhaps chemoprevention of lung cancer in high-risk individuals. The ideal agents would treat (or prevent) COPD and also decrease an individual's lung cancer risk. Given the high morbidity and mortality associated with each of these diseases, it is quite clear that we are in need of more effective early interventions.

Financial \& competing interests disclosure

The authors have no relevant affiliations or financial involvement with any organization or entity with a financial interest in or financial conflict with the subject matter or materials discussed in the manuscript. This includes employment, consultancies, honoraria, stock ownership or options, expert testimony, grants or patents received or pending, or royalties.

No writing assistance was utilized in the production of this manuscript. 


\section{References}

1 Shurin SB. Lung diseases. In: Morbidity and Mortality: 2012 Chart Book on Cardiovascular, Lung, and Blood Disease. National Heart, Lung, and Blood Institute, MD, USA, 68-91 (2012).

2 Siegel R, Naishadham MA, Jemal A. Cancer statistics, 2012. CA Cancer J. Clin. 62, 10-29 (2012).

3 Wasswa-Kintu S, Man SFP, Pare PD, Sin DD. Relationship between reduced forced expiratory volume in one second and the risk of lung cancer: a systematic review and meta-analysis. Thorax 60, 570-575 (2005).

4 De Torres JP, Mann JM, Casanova C et al. Lung cancer in patients with chronic obstructive pulmonary disease: incidence and predicting factors. Am. J. Respir. Crit. Care Med. 184, 913-919 (2011).

5 Schwartz AG. Genetic epidemiology of cigarette smoke-induced lung disease. Proc. Am. Thorac. Soc. 9(2), 22-26 (2012).

6 Rooney C, Sethi T. The epithelial cell and lung cancer: the link between chronic obstructive pulmonary disease and lung cancer. Respiration 81, 89-104 (2011).

7 Engels EA, Shen M, Chapman RS et al. Tuberculosis and subsequent risk of lung cancer in Xuanwei, China. Int. J. Cancer 124, 1183-1187 (2009).

8 Wu CY, Hu HY, Pu CY et al. Pulmonary tuberculosis increases the risk of lung cancer: a population-based cohort study. Cancer 117(3), 618-624 (2011).

9 Lande L, Peterson DD, Gogoi R et al. Association between pulmonary Mycobacterium avium complex infection and lung cancer. J. Thorac. Oncol. 7(9), 1345-1351 (2012).

10 Daley CL, Iseman M. Mycobacterium avium complex and lung cancer: chicken or egg? Both? J. Thorac. Oncol. 7(9), 1329-1330 (2012).

11 Parimon T, Chien JW, Bryson CL, McDonell $\mathrm{MB}$, Udris EM, Au DH. Inhaled corticosteroids and risk of lung cancer among patients with chronic obstructive pulmonary disease. Am. J. Respir. Crit. Care Med. 175, 712-719 (2007).

12 Veronesi G, Szabo E, DeCensi A et al. Randomized Phase II trial of inhaled budesonide versus placebo in high risk individuals with CT-screen detected lung nodules. Cancer Prev. Res. 4(1), 34-42 (2011).

13 Van Den Berg RM, Teertstra HJ, van Zandwiijk $\mathrm{N}$ et al. CT detected indeterminate pulmonary nodules in a chemoprevention trial of fluticasone. Lung Cancer 60, 57-61 (2008).

14 Keith RL. Lung cancer chemoprevention. Proc. Am. Thorac. Soc. 9(2), 52-56 (2012).

15 Ji H, Houghten AM, Mariani TJ et al. K-ras activation generates an inflammatory response in lung tumors. Oncogene 25, 2105-2112 (2006).
16 Belinsky SA, Palmisano WA, Gilliland FD et al. Aberrant promoter methylation in bronchial epithelial and sputum from current and former smokers. Cancer Res. 62, 2370-2377 (2006).

17 Qiu W, Baccarelli A, Carey A et al. Variable DNA methylation is associated with chronic obstructive pulmonary disease and lung function. Am. J. Resp. Crit. Care Med. 185(4) 373-381 (2012).

18 Mascaux C, Laes JF, Anthoine G et al. Evolution of microRNA expression during human bronchial squamous carcinogenesis. Eur. Resp. J. 33, 352-359 (2009).

19 Lee G, Walser TC, Dubinett, SM et al. Chronic inflammation, chronic obstructive pulmonary disease, and lung cancer. Curr. Opin. Pulm. Med. 15, 303-307 (2009).

20 Schwartz AG, Ruckdeschel JC. Familial lung cancer: genetic susceptibility and relationship to chronic obstructive pulmonary disease. Am. J. Respir. Crit. Care Med. 173, 16-22 (2006).

21 Galvan A, Tommaso TA. Nicotine dependence may link the $15 \mathrm{q} 25$ locus to lung cancer risk. Carcinogenesis 31(3), 331-333 (2010).

22 Karoor V, Le M, Merrick D, Fagan KA, Dempsey EC, Miller YE. Alveolar hypoxia promotes murine lung tumor growth through a VEGFR-2/EGFR-dependent mechanism. Cancer Prev. Res. 5, 1061-1071 (2012). 